changeable network of $\mathrm{Nr} 5 \mathrm{a} 2$ interactions.

Other questions are raised by this work, too. For instance, how does caerulein treatment modulate Nr5a2 function? Cobo et al. find that $\mathrm{Nr} 5 \mathrm{a} 2$ is redistributed to inflammatory-gene targets within 30 minutes of a single caerulein dose, and that expression of these targets is fully induced within one hour. This implies a rapid mechanism, possibly not dependent on increased c-Jun expression. In addition, this work does not prove that $\mathrm{Nr} 5 \mathrm{a} 2$ is required to activate the inflammatory genes that it binds - this will require comparison of mouse pancreases lacking one Nr5a2 allele with those lacking both (null), in which there is no Nr5a2. It is notable that a previous comparison of $\mathrm{Nr} 5 \mathrm{a} 2$ wild-type and null pancreases did not identify differences in inflammatory-gene expression ${ }^{9}$, potentially confirming that residual $\mathrm{Nr} 5 \mathrm{a} 2$ is needed to activate inflammatory genes.

Inflammation promotes tumour development in many tissues ${ }^{1}$, and Cobo and colleagues' study suggests that altering susceptibility to inflammation may be one way in which common single-nucleotide mutations contribute to the risk of cancer. If future work reveals that human NR5A2 mutations associated with PDAC risk reduce NR5A2 levels in the healthy pancreas, it might be possible to offset the cancer risk using anti-inflammatory drugs. A more targeted approach could take advantage of the potential for NR5A2 to bind and be activated by small organic molecules ${ }^{10,11}$. Such an activating drug might decrease the severity or frequency of pancreatitis in people with insufficient NR5A2, by raising the transcriptional activity of the residual protein.

L. Charles Murtaugh is in the Department of Human Genetics, University of Utah, Salt Lake City, Utah 84112, USA. Raymond J. MacDonald is in the Hamon Center of Regenerative Science and Medicine and the Department of Molecular Biology, University of Texas Southwestern Medical Center, Dallas, Texas 75390, USA.

e-mails:murtaugh@genetics.utah.edu; raymond.macdonald@utsouthwestern.edu

1. Grivennikov, S. I., Greten, F. R. \& Karin, M. Cell 140, 883-899 (2010)

2. Yadav, D. \& Lowenfels, A. B. Gastroenterology 144 , 1252-1261 (2013).

3. Murtaugh, L. C. \& Keefe, M. D. Annu. Rev. Physiol. 77, 229-249 (2015).

4. Shi, G. et al. Gastroenterology 136, 1368-1378 (2009).

5. Krah, N. M. et al. eLife 4, e07125 (2015)

6. von Figura, G., Morris, J. P., Wright, C. V. E. \& Hebrok, M. Gut 63, 656-664 (2014).

7. Cobo, I. et al. Nature 554, 533-537 (2018).

8. Flandez, M. et al. Gut 63, 647-655 (2014).

9. Holmstrom, S. R. et al. Genes Dev. 25, 1674-1679 (2011).

10.Lee, J. M. et al. Nature 474, 506-510 (2011).

11.Sablin, E. J. et al. J. Struct. Biol. 192, 342-348 (2015).

This article was published online on 14 February 2018.

\title{
Neurons mimicked by electronics
}

Electronic devices can currently emulate only basic functions of biological neurons. Devices called memtransistors could overcome this limitation and give rise to improvements in artificial-intelligence systems. SEE LETTER P.500

\section{DA LI \& XIAOGAN LIANG}

$\mathrm{T}$ The human brain contains billions of neurons that are linked to one another by trillions of tiny contacts called synapses. Designing electronic devices that approach this level of connectivity remains challenging, but, on page 500, Sangwan et al. ${ }^{1}$ report a breakthrough in this quest. They present a multi-terminal device called a memtransistor, which is made using a single layer of the semiconductor molybdenum disulfide $\left(\mathrm{MoS}_{2}\right)$. Such devices could enable the construction of neural-network systems that have a high degree of connectivity and that can execute complex neural functions. This envisaged network technology, if eventually created, would have a great impact on the development of hardware-based artificial intelligence (AI).

For a long time, AI was regarded as science fiction, presented in a variety of forms from humanoid robots to supercomputers. However, the past few years have witnessed rapid progress towards the realization of these fictional machines. For example, in 2016, AlphaGo became the first AI program to defeat a world champion at the game of Go - a victory that greatly increased public interest in AI research (see go.nature.com/2g9kruc).

Neural networks could enable the generation of AI systems that have unprecedented analytical and computational capabilities. However, these networks are typically constructed using electronic components called complementary 


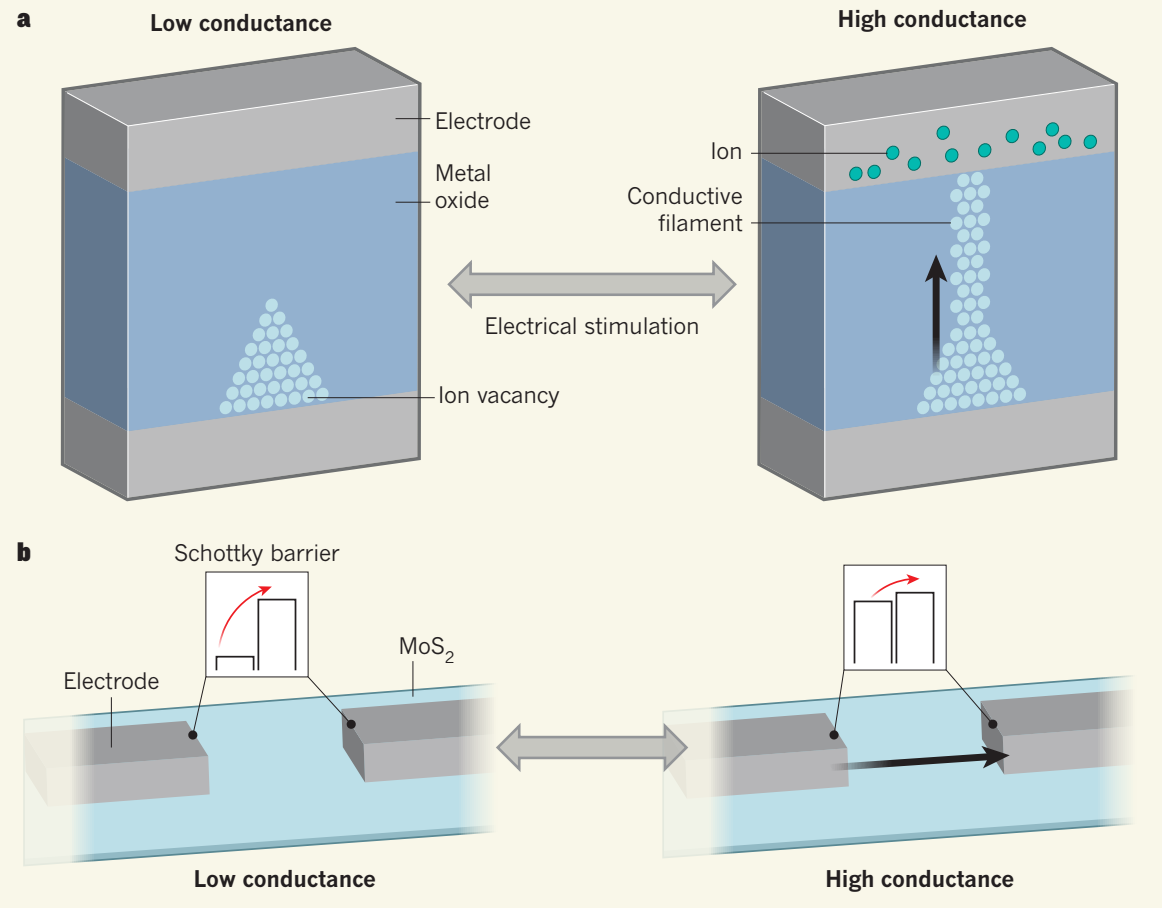

Figure 1 | Neuron-inspired electronic switches. a, Electronic devices known as memristors can emulate basic functions of biological neurons. Conventional memristors consist of a metal-oxide material and two electrodes ${ }^{3,4}$. External electrical stimulation can cause the device to switch between low- and high-conductance states, which are determined by the distribution of internal ions or ion vacancies. In particular, in the high-conductance state, there is a conductive filament of ion vacancies that enables the flow of electric charge ${ }^{5}$ (black arrow). b, Sangwan et al. ${ }^{1}$ report a device called a memtransistor that provides a platform potentially capable of carrying out complex neural functions. The device comprises a single layer of molybdenum disulfide $\left(\mathrm{MoS}_{2}\right)$ and two or more electrodes. In the low-conductance state, there are high energy barriers called Schottky barriers that charged particles must overcome (red arrow) to travel between electrodes. By contrast, in the high-conductance state, the Schottky barriers are low, which facilitates the flow of electric charge.

metal-oxide-semiconductor (CMOS) devices, which need to be intensively programmed using software. This results in formidable computing complexity and high (megawattlevel) power consumption ${ }^{2}$. These drawbacks severely hinder the practical application of CMOS-based neural-network systems for power-hungry computing tasks, such as multimedia streaming and the control of soft and flexible robots.

To tackle these challenges, researchers are exploring alternative device architectures and principles to those currently used, and different ways to combine (integrate) devices. The aim is to realize energy-efficient AI processes at the hardware level. One approach is to create electronic switches capable of emulating the mechanism that governs signal transmission in the human brain. This mechanism involves changes in the strength of synaptic connections between neurons that correlate with learning and memory ${ }^{2}$. Electronic components known as memristors have become promising candidates for such an application.

Conventional memristors are made from transition-metal oxides ${ }^{3,4}$. In such devices, external electrical stimulation can cause

a substantial change in conductance the device switches between low- and high-conductance states. These states are determined by the distribution of internal ions or ion vacancies, and the switching of the states is attributed to the formation and rupture of a conductive filament that connects two electrodes ${ }^{5}$ (Fig. 1a).

A limitation of this filament-based operation scheme is that the memristor can have only two terminals (connections

\section{"We earnestly hope that, in the near future, small-scale memtransistor networks will be constructed."} between the metaloxide material and the electrodes). Although neural networks consisting of two-terminal memristors can execute basic neural functions ${ }^{5}$, the human brain contains many more synapses than neurons. This observation suggests that multiterminal devices based on a non-filament operation principle are needed for carrying out complex neural functions.

The conductance state of Sangwan and colleagues' $\mathrm{MoS}_{2}$ memtransistor is mainly determined by the heights of energy barriers called Schottky barriers that charged particles must overcome to travel between electrodes (Fig. 1b). External voltages can be used to tune the heights of these barriers and switch between conductance states.

This state-switching principle distinguishes $\mathrm{MoS}_{2}$ memtransistors from filament-based memristors. In particular, it provides a platform for creating multi-terminal circuits capable of mimicking biological neurons that have many synaptic connections. The authors created a memtransistor that has six electrodes to demonstrate this capability (see Figure $4 \mathrm{~b}$ of the paper ${ }^{1}$ ). Because the conductance state between any two electrodes is controlled by voltage-tuned Schottky barriers instead of by filament formation and rupture, the states can be easily tuned by varying the heights of individual barriers. In addition, because the memtransistor uses only a single layer of $\mathrm{MoS}_{2}$, it is extremely thin - which means that the states can also be tuned using an electrode underneath the device, potentially enabling a larger range of states to be accessible.

Despite the benefits offered by the authors' memtransistor, various challenges must be overcome before such devices can be used to construct working neural networks. For example, industry currently lacks the ability to manufacture large areas of highly uniform single-layer $\mathrm{MoS}_{2}$. Furthermore, modelling work involving molecular dynamics is needed to fully understand the atomic-level processes responsible for the device's switching characteristics.

Sangwan and colleagues' work is still at the device level, and the authors have not provided a realistic design for a complete neural-network system composed of $\mathrm{MoS}_{2}$ memtransistors. We earnestly hope that, in the near future, small-scale memtransistor networks will be constructed and used to demonstrate basic neural functions. Such functions could include: pattern classification; a type of learning known as dictionary learning; and feature extraction, in which the number of resources required to describe a large set of data is reduced. Finally, it should be pointed out that biological neurons are arranged in 3D space. This fact could motivate studies that address the challenges associated with the $3 \mathrm{D}$ integration of multi-terminal memtransistors.

\section{Da Li and Xiaogan Liang are in the}

Mechanical Engineering Department,

University of Michigan, Ann Arbor,

Michigan 48109, USA.

e-mail:xiaoganl@umich.edu

1. Sangwan, V. K. et al. Nature 554, 500-504 (2018)

2. Prezioso, M. et al. Nature 521, 61-64 (2015).

3. Williams, R. S. IEEE Spectrum 45, 29-35 (2008).

4. Strukov, D. B., Snider, G. S., Stewart, D. R. \& Williams, R. S. Nature 453, 80-83 (2008).

5. Kim, S., Choi, S.-H. \& Lu, W. ACS Nano 8, 2369-2376 (2014). 